\title{
The Putative Atypical Antipsychotic Drug Amperozide Preferentially Increases $c$-fos Expression in Rat Medial Prefrontal Cortex and Lateral Septum
}

George G. Nomikos, M.D., Ph.D., Chui-Se Tham, B.Sc., H. Christian Fibiger, Ph.D., and

Torgny H. Svensson, M.D., Ph.D.

The effects of acute, systemic administration of the putative atypical antipsychotic drug amperozide on c-fos expression in the rat forebrain were studied by means of Fos immunohistochemistry. Amperozide significantly increased the number of Fos-immunoreactive nuclei in the medial prefrontal cortex and the lateral septum but not in the nucleus accumbens (shell or core), the striatum, or the amygdala. With the exception of the nucleus accumbensshell, where amperozide failed to produce statistically significant increases, the regional distribution of Fos immunoreactivity following amperozide was similar to that induced by atypical, but not by typical, antipsychotic drugs. In addition, after amperozide the number of Fos-positive nuclei was higher in the nucleus accumbens than in the dorsolateral striatum, a characteristic that is common to all known atypical antipsychotic agents.

[Neuropsychopharmacology 17:197-201, 1997]

(C) 1997 American College of Neuropsychopharmacology. Published by Elsevier Science Inc.
KEY WORDS: Dopamine; Immediate early genes; Nucleus accumbens; Serotonin; Striatum

Amperozide is a new, putative atypical antipsychotic drug characterized by a high affinity for serotonin (5$\mathrm{HT})_{2}$ receptors, and relatively low to moderate affinity for dopamine (DA) receptors $\left(\mathrm{D}_{1}\right.$ or $\left.\mathrm{D}_{2}\right)$, $\alpha$-adrenoceptors and other serotonergic receptors (Meltzer et al. 1989, 1992; Roth et al. 1992; Svartengren and Celander 1994; Svartengren and Simonsson 1990). The results of behavioral, biochemical, and electrophysiological stud-

From Karolinska lnstitutet, Department of Physiology and Pharmacology, Division of Pharmacology, Stockholm, Sweden (GGN, THS), and the Division of Neurological Sciences, Department of Psychiatry, University of British Columbia, Vancouver B.C., Canada (C-ST, HCF).

Address correspondence to: Dr. G.G. Nomikos, Karolinska Institutet, Department of Physiology and Pharmacology, Division of Pharmacology, S-171 77 Stockholm, Sweden.

Received August 7, 1996; revised November 22, 1996; accepted February 21, 1997. ies suggest that amperozide exhibits a limbic mode of action (Grenhoff et al. 1990; Gustafsson and Christensson 1990; Pettersson et al. 1990). Recently, Pehek et al. (1993) and Nomikos et al. (1994) using in vivo microdialysis, and Marcus et al. (1996) using in vivo voltammetry, showed that like clozapine, amperozide increases DA release in the medial prefrontal cortex (mPFC) to a greater extent than in the nucleus accumbens (NAcc) or the striatum, and that it selectively enhances DA release in the shell subdivision of the NAcc, which is largely associated with limbic functions, but not in the motorically-related core subdivision of the NAcc. In addition, open clinical trials have suggested that amperozide may have antipsychotic actions, affecting both positive and negative symptoms, with a very low propensity to induce extrapyramidal side effects (Axelsson et al. 1991; Björk et al. 1992).

Immediate early genes, such as $c$-fos, have proven to be useful markers of changes in neuronal activity, and 
recently Fos immunohistochemistry has been used to examine the effects of antipsychotic drugs in the brain (Deutch et al. 1992; Dragunow et al. 1990; Guo et al. 1995; MacGibbon et al. 1994; Miller et al. 1990; Nguyen et al. 1992; Robertson and Fibiger 1992; Robertson et al. 1994). These studies have shown that typical and atypical antipsychotic drugs differentially affect $c$-fos expression in various regions of the brain. Thus, haloperidol induces $c$-fos, expression in the NAcc, the lateral septum and the striatum, whereas clozapine increases $c$-fos expression in the NAcc, the lateral septal nucleus, and the mPFC. In fact, based on calculations of the difference between the number of neurons displaying Fos-like immunoreactivity (FLI) in the NAcc and that in the dorsolateral striatum in response to several antipsychotic drugs, an index of atypicality has been proposed that is positive for atypical antipsychotics and negative for classical neuroleptics (Robertson et al. 1994). It is conceivable, therefore, that the regional specificity of antipsychotic-mediated immediately early gene expression may reflect different patterns of neuronal responses and consequently differences in the clinical profile between the typical and atypical antipsychotic drugs.

The purpose of the present study was to study the effects of acute, systemic administration of amperozide on the number and anatomical distribution of Fos-positive neurons in the basal ganglia and various limbic regions, particularly in the mPFC, the NAcc (shell and core), the striatum, the lateral septum, and the amygdala.

\section{MATERIALS AND METHODS}

Male Wistar rats weighing 300-400 g were used. All rats were housed individually for at least one week before the experiment and were handled for 5-10 minutes daily the last two days. Amperozide hydrochloride (Pharmacia Leo Therapeutics AB) was dissolved in $0.9 \% \mathrm{NaCl}$ (saline) and injected subcutaneously (s.c.) in doses of 5.0 or $10.0 \mathrm{mg} / \mathrm{kg}$ (11.4 or $22.8 \mu \mathrm{mol} / \mathrm{kg}$ ) in the neck; control animals were injected with saline $(1.0 \mathrm{ml} /$ $\mathrm{kg}$ ). Two hours after saline or amperozide injection, all animals were deeply anesthetized with pentobarbital $(100 \mathrm{mg} / \mathrm{kg}$ or $0.40 \mathrm{mmol} / \mathrm{kg}$, intraperitoneally) and perfused transcardially with saline $(200 \mathrm{ml})$ followed by $4 \%$ paraformaldehyde $(300 \mathrm{ml})$ in phosphate-buffered $(0.1$ $\mathrm{M})$ saline (PBS). Each brain was removed immediately after perfusion and placed in fixative for at least 12 hours.

Fos immunohistochemistry was performed as described by Robertson and Fibiger (1992) and Chergui et al. (1996). After the postfixative period, $30 \mu \mathrm{m}$ coronal sections were cut from each brain using a vibratome. Sections were washed three times with $0.02 \mathrm{M}$ PBS and then incubated in PBS containing 0.3\% hydrogen peroxide. Sections were then washed three times in PBS containing $0.3 \%$ Triton- $X, 0.02 \%$ sodium azide and Fos pri- mary antibody (sheep polyclonal antibody, Cambridge Research Biochemicals OA-11-823) diluted 1:2000 for 48 hours. The sections were then washed three times with PBS and incubated with a biotinylated rabbit anti-sheep secondary antibody (Vector Laboratories) diluted 1:500 for 1 hour. The sections were washed three times with PBS and incubated for 1 hour with PBS containing 0.3\% Triton-X and $0.5 \%$ avidin-biotinylated horseradish peroxidase complex (Vector Laboratories). After three washes in PBS the sections were rinsed in $0.1 \mathrm{M}$ acetate buffer ( $\mathrm{pH}$ 6.0). The reaction was visualized with the glucose oxidase-3,3'-diaminobenzidine-nickel method. The reaction was terminated by washing in PBS, and the sections were mounted on chrome-alum coated slides, dehydrated and prepared for microscopic observation.

The number of Fos-like-immunoreactive nuclei was counted under microscopic control within a grid placed over the MPFC, the NAcc, the medial and lateral striatum, the lateral septum, and the basolateral amygdala as illustrated in previous studies (Robertson and Fibiger 1992-Fig. 1; Brown et al. 1992-Figure 1). Specifically, the anterior-posterior coordinates (measured from bregma according to Paxinos and Watson 1986) of sections in which Fos-positive nuclei were counted were $+2.7,+1.2$, $+1.0,+0.7$ and $-2.8 \mathrm{~mm}$ for the mPFC, the NAcc, the striatum, the septum, and the amygdala, respectively. The size of the grid was $500 \times 500 \mu \mathrm{m}$ and labelled cells were counted at $\times 250$ magnification. In addition, Fospositive cells were counted in the shell and core subdivisions of the NAcc as shown in the study by Chergui et al. (1996-Figure 2) and by Deutch et al. (1992-Figure 1). Although no marker such as calcium-binding protein was used to distinguish between these two parts of the NAcc, caution was taken to avoid overlap of the sampled areas for each structure by placing the grid $(300 \times 300 \mu \mathrm{m})$ close to the anterior commissure for the core and between core and septum for the shell. For each brain, in both hemispheres and each structure, cells were counted by two observers in three slices and the mean value of 12 measurements was calculated.

A one-way analysis of variance (ANOVA) was performed on the cell count data for the two doses of amperozide and vehicle from each brain region. If the ANOVA was significant, multiple comparisons were performed using the Newman-Keuls test.

\section{RESULTS}

Both doses of amperozide significantly increased the number of Fos-positive nuclei in the mPFC $(p=0.011$ and $p=0.017$ for the 5.0 and $10.0 \mathrm{mg} / \mathrm{kg}$ dose, respectively) and the lateral septum ( $p=0.011$ for either the 5.0 or the $10.0 \mathrm{mg} / \mathrm{kg}$ dose; Figures 1 and 2). In the mPFC, the amperozide-induced Fos-positive cells were evenly distributed among the cortical layers, although rela- 
tively fewer cells were found in the superficial compared to the deep layers. Amperozide tended to produce dose-related increases in FLI in the NAcc (shell), although this did not reach statistical significance. Similarly, amperozide did not significantly influence FLI in the NAcc (core), the striatum (dorsolateral or medial), or the amygdala (Figure 2). The number of Fos-positive nuclei was also not significantly affected by amperozide when a larger part of the NAcc was considered (data not shown), as in the study by Robertson and Fibiger (1992).
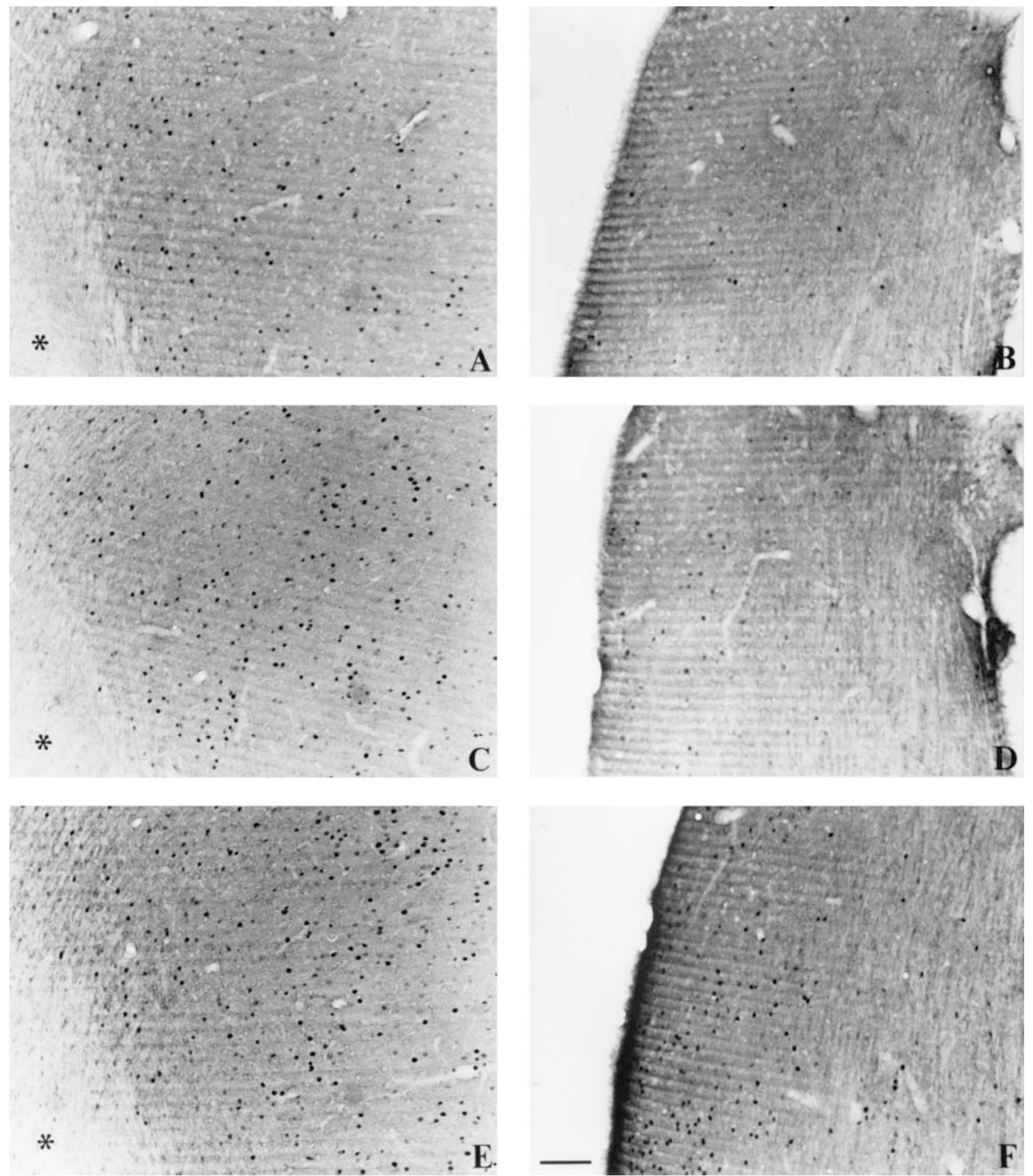

Figure 1. Low-magnification $(\times 100)$ photomicrographs illustrating Fos-like immunoreactivity in the medial prefrontal cortex $(\mathrm{A}, \mathrm{C}, \mathrm{E}$; stars indicate the forceps minor, corpus callosum) or the lateral septum $(\mathrm{B}, \mathrm{D}, \mathrm{F})$ of the rat in response to saline $(\mathrm{A}, \mathrm{B})$, the $5.0(\mathrm{C}, \mathrm{D})$, or the $10.0(\mathrm{E}, \mathrm{F}) \mathrm{mg} / \mathrm{kg}$ dose of amperozide. Scale bar $=100 \mu \mathrm{m}$. 


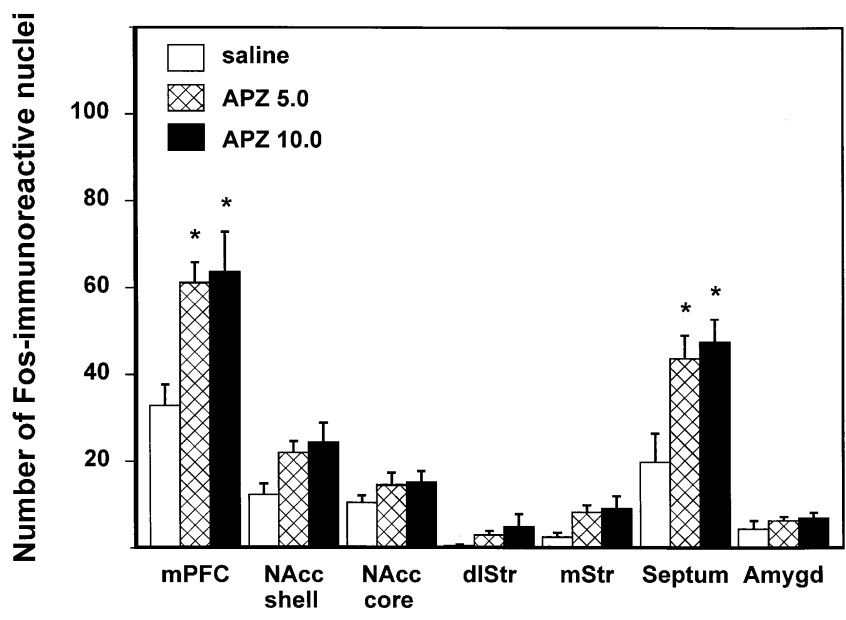

Figure 2. Effect of saline $(\mathrm{N}=5)$ or amperozide (APZ; doses in $\mathrm{mg} / \mathrm{kg} ; \mathrm{N}=6$ for each dose) on the number of Foslike immunoreactive cells in the medial prefrontal cortex (mPFC), the shell or the core subdivision of the nucleus accumbens (NAcc), the dorsomedial striatum (dlStr), the medial striatum (mStr), the lateral septum and the basolateral amygdala (Amygd) of the rat. Bars indicate the mean $(+\mathrm{SEM})$ number of Fos-like immunoreactive nuclei. ${ }^{*} p<$ 0.05 compared to saline.

However, the calculated atypical index, i.e., the number of Fos-positive neurons in the NAcc minus the number in the dorsolateral striatum after correction for the injection procedure (Robertson et al. 1994), was positive for both the $5.0(+9.5)$ and the $10.0(+15.5) \mathrm{mg} / \mathrm{kg}$ dose of amperozide; statistical analysis revealed that the calculated atypical index in response to the high dose of amperozide was significantly $(p=0.036)$ different from that produced by the vehicle injection.

\section{DISCUSSION}

Like clozapine, amperozide significantly increased the number of Fos-immunoreactive nuclei in the mPFC and the lateral septum but not in the dorsolateral striatum. On the other hand, unlike clozapine, amperozide did not significantly influence FLI in the medial striatum or the NAcc. However, the index of atypicality calculated as the difference between the number of neurons displaying FLI in the NAcc and the dorsolateral striatum was positive for both doses, indicating that by this criterion amperozide can be classified as an atypical antipsychotic drug.

Based on in vivo receptor binding data (Meltzer et al. 1992; Svartengren and Celander 1994), the occupancy of $5-\mathrm{HT}_{2}$ receptors with the doses of amperozide used in the present study can be estimated to be high $(>75 \%)$, whereas the $\mathrm{D}_{2}$ receptor occupancy is probably low $(<25 \%)$. Thus, the amperozide-induced $c$-fos expression in the MPFC and the lateral septum may be related to its potent antagonist actions at $5-\mathrm{HT}_{2}$ receptors. However, previous studies have shown that ritanserin, a potent $5-\mathrm{HT}_{2}$ receptor antagonist, fails to induce $c$-fos expression in the relevant brain regions (Guo et al. 1995; Wan et al. 1995). These results suggest that antagonist actions of amperozide at $5-\mathrm{HT}_{2}$ receptors do not contribute to its effects on $c$-fos expression. Moreover, in a study by Guo et al. (1995), combined administration of ritanserin with haloperidol failed to mimic the pattern of $c$-fos expression produced by clozapine in the forebrain and particularly in the MPFC, although a similar combination of ritanserin with a low dose of raclopride, a selective $D_{2}$ receptor antagonist, results in a higher DA release in this brain region than raclopride alone (Andersson et al. 1995). In this regard, it is striking that the regional distribution of FLI produced by amperozide and clozapine, is similar to the pattern of their DA releasing effect in the forebrain, i.e., a more pronounced action in the mPFC than in subcortical dopaminergic regions (Moghaddam and Bunney 1990; Pehek et al. 1993; Nomikos et al. 1994; Robertson and Fibiger 1992).

Both doses of amperozide significantly increased $c$-fos expression in the lateral septum. The fact that almost all the clinically effective antipsychotic drugs, both typical and atypical, increase the number of Fosimmunoreactive neurons in the lateral septal nucleus (MacGibbon et al. 1994; Robertson and Fibiger 1992; Robertson et al. 1994; Wan et al. 1995) suggests that this part of the limbic system may be a significant part of the circuit mediating antipsychotic drug action. On the other hand, in contrast to all other antipsychotic agents tested to date (Deutch et al. 1992; Robertson et al. 1994; Robertson and Fibiger 1996), amperozide failed to significantly increase $c$-fos expression in the shell part of the NAcc. In this aspect, amperozide shows a unique profile with the implication that its putative antipsychotic action may be associated with actions in the brain that do not involve the shell of the NAcc. If future clinical studies indicate that amperozide is indeed a clinically effective antipsychotic, then it will be the first such agent that fails to significantly increase $c$-fos expression in the NAcc. This would have important implications regarding the brain regions that play a role in the therapeutic effects of antipsychotic drugs.

\section{ACKNOWLEDGMENTS}

This work was supported by the Swedish Medical Research Council (projects 04747 and 11026), the Swedish Society of Medicine, the Karolinska Institute, and LEO's Research Foundation and Janssen Pharmaceutica, Beerse, Belgium. The authors thank Mrs. Anna Malmerfelt for help with the counting of cells and for preparing the photomicrographs. 


\section{REFERENCES}

Andersson JL, Nomikos GG, Marcus M, Hertel P, Mathé JM, Svensson TH (1995): Ritanserin potentiates the stimulatory effects of raclopride on neuronal activity and dopamine release selectively in the mesolimbic dopaminergic activity. N-S Arch Pharmacol 352:374-385

Axelsson R, Nilsson A, Christensson E, Björk A (1991): Effects of amperozide in schizophrenia. Psychopharmacology 104:287-292

Björk A, Bergman I, Gustavsson G (1992): Amperozide in the treatment of schizophrenic patients. A preliminary report. In Meltzer HY (ed), Novel antipsychotic drugs. Raven, New York, pp 45-57

Brown EE, Robertson GS, Fibiger HC (1992): Evidence for conditional neuronal activation following exposure to a cocaine-paired environment: role of forebrain limbic structures. J Neurosci 12:4112-4121

Chergui K, Nomikos GG, Mathé JM, Gonon F, Svensson TH (1996): Burst stimulation of the medial forebrain bundle selectively increases Fos-like immunoreactivity in the limbic forebrain of the rat. Neuroscience 72:141-156

Deutch AY, Lee MC, Iadarola MJ (1992): Regionally specific effects of atypical antipsychotic drugs on striatal Fos expression: the nucleus accumbens shell as a locus of antipsychotic action. Molec Cell Neurosci 3:332-341

Dragunow M, Robertson GS, Faul RLM, Robertson HA, Jansen K (1990): D2 dopamine receptor antagonists induce Fos and related proteins in rat striatal neurons. Neuroscience 37:287-294

Grenhoff J, Tung C, Ugedo L, Svensson TH (1990): Effects of amperozide, a putative antipsychotic drug, on rat midbrain dopamine neurons recorded in vivo. Pharmacol Toxicol 66 (suppl 1):29-33

Guo N, Klitenick MA, Tham C-S, Fibiger HC (1995): Receptor mechanisms mediating clozapine-induced c-fos expression in the forebrain. Neuroscience 65:747-756

Gustafsson B, Christensson E (1990): Amperozide-a new putatively antipsychotic drug with a limbic mode of action on dopamine mediated behavior. Pharmacol Toxicol 66 (suppl. 1):12-17

MacGibbon GA, Lawlor PA, Bravo R, Dragunow M (1994): Clozapine and haloperidol produce a differential pattern of immediate early gene expression in rat caudateputamen, nucleus accumbens, lateral septum and islands of Calleja. Molec Brain Res 23:21-32

Marcus MM, Nomikos GG, Svensson TH (1996): Differential actions of typical and atypical antipsychotic drugs on dopamine release in the core and shell of the nucleus accumbens. Eur Neuropsychopharmacol 6:29-38

Meltzer HY, Matsubara S, Lee JC (1989): Classification of typical and atypical antipsychotic drugs on the basis of dopamine D-1, D-2 and serotonin2 pKi values. J Pharmacol Exp Ther 251:238-246
Meltzer HY, Zhang Y, Stockmeier CA (1992): Effects of amperozide on rat cortical 5-HT2 and striatal and limbic dopamine D2 receptor occupancy: implications for antipsychotic action. Eur J Pharmacol 216:67-71

Miller JC (1990) Induction of c-fos mRNA expression in rat striatum by neuroleptic drugs. J Neurochem 54:1453-1455

Moghaddam B, Bunney BS (1990): Acute effects of typical and atypical antipsychotic drugs on the release of dopamine from prefrontal cortex, nucleus accumbens, and striatum of the rat: an in vivo microdialysis study. J Neurochem 54:1755-1760

Nguyen TY, Kosofsky BE, Birnbaum R, Cohen BM, Hyman SE (1992): Differential expression of c-fos and zif268 in rat striatum, after haloperidol, clozapine and amphetamine. Proc Natl Acad Sci U.S.A. 89:4270-4274

Nomikos GG, Iurlo M, Andersson JL, Kimura K, Svensson TH (1994): Systemic administration of amperozide, a new atypical antipsychotic drug, preferentially increases dopamine release in the rat medial prefrontal cortex. Psychopharmacology 115:147-156

Paxinos G, Watson C (1986): The rat brain in stereotaxic coordinates, 2nd ed. New York, Academic Press

Pehek EA, Melzer HY, Yamamoto BK (1993): The atypical antipsychotic drug amperozide enhances rat cortical and striatal dopamine efflux. Eur J Pharmacol 240:107-109

Pettersson G, Johannessen K, Hulthe P, Engel JA (1990): Effect of amperozide on the synthesis and turnover of monoamines in rat brain. Pharmacol Toxicol 66 (suppl. 1):40-44

Robertson GS, Fibiger HC (1992): Neuroleptics increase c-fos expression in the forebrain: contrasting effects of haloperidol and clozapine. Neuroscience 46:315-328

Robertson GS, Matsubura H, Fibiger HC (1994): Induction patterns of Fos-like immunoreactivity in the forebrain as predictors of atypical antipsychotic activity. J Pharmacol Exp Ther 271:1058-1066

Robertson GS, Fibiger HC (1996): Effects of olanzapine on regional c-fos expression in the rat forebrain. Neuropsychopharmacol 14:105-110

Roth BL, Ciaranello RD, Meltzer HY (1992): Binding of typical and atypical antipsychotic agents to transiently expressed 5-HT1C receptors. J Pharmacol Exp Ther 260: 1361-1365

Svartengren J, Celander M (1994): The limbic functional selectivity of amperozide is not mediated by dopamine D2 receptors as assessed by in vitro and in vivo binding. Eur J Pharmacol 254:73-81

Svartengren, J, Simonsson P (1990): Receptor binding properties of amperozide. Pharmocol Toxicol 66 (suppl 1):8-11

Wan W, Ennulat DJ, Cohen BM (1995): Acute administration of typical and atypical antipsychotic drugs induces distinctive patterns of Fos expression in the rat forebrain. Brain Res 688:95-104 